\title{
Finite-Temperature Transition into a Power-Law Spin Phase with an Extensive Zero-Point Entropy
}

\author{
P. Chandra \\ NEC Research Institute, 4 Independence Way, Princeton, NJ 08540. \\ P. Coleman and L.B. Ioffe \\ Serin Physics Laboratory, Rutgers University, P.O. Box 849, Piscataway, NJ 08854.
}

\begin{abstract}
We introduce an $x y$ generalization of the frustrated Ising model on a triangular lattice. The presence of continuous degrees of freedom stabilizes a finite-temperature spin state with power-law discrete spin correlations and an extensive zero-point entropy. In this phase, the unquenched degrees of freedom can be described by a fluctuating surface with logarithmic height correlations. Finite-size Monte Carlo simulations have been used to characterize the exponents of the transition and the dynamics of the low-temperature phase.
\end{abstract}

Typeset using REVTEX 


\section{INTRODUCTION}

The possibility of spin systems with a residual zero-point entropy has existed since Wannier's study of the two-dimensional Ising triangular antiferromagnet; 1 here the frustration leads to a zero-temperature $(T=0)$ critical point characterized by an extensive entropy $S_{0}=0.338$. The associated unquenched degrees of freedom can be described by a fluctuating surface with logarithmic height correlations, 目 3 in discrete spin models the weak nature of the long-range entropic interactions between height defects forbids the existence of this critical phase at $T \neq 0$. Can a phase transition into a state with a zero-point entropy exist at finite temperatures? This issue was first raised by Anderson 1 in his study of spinels, where he noted that a [111] projection of this structure corresponds to a planar kagomé net. Anderson suggested that the ground-state manifold of Heisenberg spins on such frustrated lattices would be highly degenerate, and thus that long-range magnetic order would be suppressed due to the residual entropy at finite temperatures. power-law discrete spin correlations at finite temperatures in contrast to the exponential and constant Ising correlations associated with a paramagnet and an ordered phase; it is thus natural to call this state a "classical spin liquid".

There now exists an experimental realization of Anderson's model: the magnetoplumbite $\mathrm{SrCr}_{8-x} \mathrm{Ga}_{4+x} \mathrm{O}_{19}(S C G O(x))$, whose magnetic properties are attributed to planes of antiferromagnetically-coupled chromium atoms on a kagomé lattice. tron scattering $\theta^{6}$ and specific heat measurements on $S C G O(x)$ are consistent with antiferromagnetic order, the observed spin correlation length is only a few lattice spacings. exist many other anomalous magnetic materials that, like $S C G O(x)$, do not display conventional long-range spin order 19 Most of these experimental systems have physical structures that impose strong constraints on the low-temperature configurations of their continuous spins, allowing them to be identified with the ground-states of an associated discrete model on the same lattice. Following the spirit of Anderson's original suggestion, 1 we show that an interplay between continuous and discrete degrees of freedom can enhance the defect-defect 
interaction in the associated fluctuating surface, thus stabilizing a finite-temperature critical point associated with a zero-point entropy.

\section{THE DISCRETE MODEL}

The two-dimensional Ising antiferromagnet with nearest-neighbor interactions on the triangular lattice is the simplest frustrated spin system; it does not order even at zero temperature but has power-law spin correlations in contrast to a conventional paramagnet. 2 The geometry of this lattice does not permit the minimization of each bond energetically and thus even at $T=0$ there exist parallel and antiparallel nearest-neighbor spin pairs which we denote as antiferromagnetic (AFM) and ferromagnetic (FM) bonds for convenience. The energy of each triangle is minimized if it contains one and only one ferromagnetic bond. The number of spin configurations that satisfy this plaquette constraint scales exponentially with the site number; the resulting $T=0$ manifold has an extensive entropy and can be fully explored from a given starting state by flipping spins with equal numbers of AFM and FM bonds (Figure 1). If a spin with four AFM bonds is reversed there result two "bad" triangles that violate the constraint (Figure 2). These two "defects" can be separated to arbitrary distance at no additional cost, despite the fact that in doing so all the spins along the line linking them are reversed (Figure 3). The situation is similar to that in the $1 d$ Ising ferromagnet where a flip of a single spin creates two "bad" bonds. These defects can move freely along the chain by reversing all the spins between

them; since no more "bad" bonds appear in this process their energy cost is independent of their relative positions. In the triangular case the analogous separation of two "bad" triangles does not lead to the formation of more "bad" plaquettes, so that their energy cost is also finite in the thermodynamic limit. At $T=0$ the creation of these defects is forbidden, there is no length-scale in the problem and the spin correlations are power-law. However at nonzero temperatures defect formation is favored entropically, and the spin correlations become exponential with a correlation length determined by the defect density. 
We contrast this situation with that of the $2 d$ Ising ferromagnet where the minimum energy cost associated with two defects at a separation $L$ scales like $E \sim L^{\frac{1}{2}}$; here defect formation is suppressed until a finite temperature.

We can characterize each spin state by its spatial configuration of antiferromagnetic bonds. At $T=0$ the plaquette constraint ensures that each triangle has exactly two AFM bonds; each spin state can thus be mapped onto a unique tiling of the plane by rhombi which can also be viewed as a planar projection of a surface in three-dimensions (Figure 4). Quantitatively this mapping between the spins $\left\{s_{i}\right\}$ and the heights $\left\{h_{i}\right\}$ associated with the surface is defined by the expression

$$
\left(h_{i}-h_{j}\right)=\frac{1}{2}\left(3 \vec{s}_{i} \cdot \vec{s}_{j}+1\right)
$$

for sites $i$ and $j$ as shown in Figure 4.If the underlying spin configuration satisfies the plaquette constraint, the sum of the height differences as defined in (1) around any closed path will be exactly zero (Figure 5a) and the height is a uniquely defined function. By contrast, a similar sum determined along a loop enclosing a "bad" triangle (see Figure 5b ) results in a finite height difference which, in analogy with conventional elasticity theory, we denote as the Burger's vector; the underlying spin configuration determines its numerical value to be a multiple of six. Defects in the surface appear because the height is now a multivalued function whose branching points correspond to triangles that violate the constraint.

The surface representation provides a very convenient description of the full ground-state manifold; in this language the spin flips associated with a zero energy cost correspond to the addition/subtraction of elementary cubes. Thus the allowed $T=0$ spin configurations are mapped onto a free fluctuating surface whose behavior at finite temperatures and long length-scales is characterized by the free energy density

$$
F=\frac{1}{2} \eta T(\nabla h)^{2}
$$

where $\eta T$ is the stiffness associated with the surface tension of the membrane. Equation (2) leads to logarithmic height correlations of the form 


$$
\left\langle\left(h_{i}-h_{j}\right)^{2}\right\rangle=\frac{1}{\eta \pi} \ln \frac{r_{i j}}{a}
$$

where $a$ is the cutoff imposed by the lattice. Since the spins and the heights on any sites $i$ and $j$ are related by the expression

$$
s_{i} \cdot s_{j}=(-1)^{\left\{h_{i}-h_{j}\right\}}
$$

the spin-spin correlation function at large distances is power-law

$$
\left\langle s_{i} \cdot s_{j}\right\rangle=\exp -\frac{\pi^{2}}{2}\left\langle\left(h_{i}-h_{j}\right)^{2}\right\rangle=\left(\frac{a}{r_{i j}}\right)^{\frac{\pi}{2 \eta}}
$$

which follows from the free energy density (2). For the $T \rightarrow 0$ Ising model with nearestneighbor interactions that we have considered above, the correlation function (5) is known from the exact solution目边 and results in a stiffness coefficient

$$
\eta_{I \operatorname{sing}}=\frac{\pi}{9}
$$

for the associated free surface. In principle, a generalized spin model on the triangular lattice could lead to a different numerical value of $\eta$, which is determined by the specific form of the spin-spin interaction.

The free energy density (2) leads to a defect energy that scales logarithmically with system size at any finite temperature. The resulting free energy associated with an isolated defect is

$$
F_{\text {defect }}=E_{0}-2 T\left\{\left(\frac{b^{2} \eta}{8 \pi}\right)-1\right\} \ln L
$$

where $E_{0}$ is the core energy and the last term in (7) is the entropy associated with the random location of the defect on the surface. This defect free energy is logarithmically large if the coefficient of the temperature in (7) is positive; for the case here where the minimum Burger's vector $b_{\min }=6$, the critical value for $\eta$ is

$$
\eta_{c_{1}}=\frac{8 \pi}{b^{2}}=\frac{2 \pi}{9}
$$


Binding of the surface defects 10 occurs for $\eta \geq \eta_{c_{1}}$, and we note that $\eta_{\text {Ising }}<\eta_{c_{1}}$ consistent with the known presence of exponential spin correlations at finite temperatures.

In the previous discussion we have implicitly assumed the irrelevance of terms in the free energy density that favor a particular surface orientation. These contributions should be periodic in the height and thus have a general form

$$
F_{r}=\sum_{n} V_{n} \cos 2 \pi n h
$$

where the most "dangerous" term is the case $n=1$ with dimension $[L]^{2-\frac{\eta}{\pi}}$. Thus for a stiffness constant larger than

$$
\eta_{c_{2}}=\frac{\pi}{2}
$$

the surface no longer fluctuates on long length-scales but remains essentally flat 11

The possibility of a fluctuating defect-free surface at finite temperatures then exists for values of the stiffness coefficient in the region

$$
\eta_{c_{1}}>\eta>\eta_{c_{2}}
$$

where defects are bound but the surface is not spontaneously oriented. In this window (11) the correlations of the discrete spins are power-law in contrast to their exponential and constant behavior for $\eta<\eta_{c_{1}}$ and $\eta>\eta_{c_{2}}$ respectively (see Figure 6). We note that this possibility for spin liquid behavior exists because the minimum Burger's vector associated with the underlying spin model is $b_{\text {min }}=6$, which is twice that allowed for the pure surface problem. The value of the stiffness constant associated with the nearest-neighbor Ising model is less than the critical value required for defect-binding, consistent with the absence of power-law Ising spin correlations at any finite temperature. We would like to construct a generalization of this model with a spin-spin interaction that leads to an enhanced stiffness coefficient. In this paper, we have introduced additional continuous degrees of freedom and show that their interaction with the Ising variables leads to the stability of a classical spin liquid phase at finite temperatures. 


\section{THE GENERALIZED SPIN MODEL}

The simplest frustrated model that has both Ising and continuous degrees of freedom is described by the generalized $x y$ Hamiltonian

$$
H=\frac{1}{2} \sum_{i j} f\left(\Theta_{i}-\Theta_{j}\right)
$$

on a triangular lattice. We specify the periodic function $f\left(\Theta_{i}-\Theta_{j}\right)$ so that in the groundstate configurations the spins are nearly collinear with angular orientations described by

$$
\Theta_{i}=\pi \sigma_{i}+\theta_{i}
$$

where $\sigma_{i}$ and $\Theta_{i}$ are the Ising and the continuous variables respectively. The angular deviation between spin orientations is small on short length-scales (i.e. $\left|\theta_{i}-\theta_{j}\right|<<\pi$ where $i$ and $j$ are nearest-neighbor sites) but can be substantial at large distances. We tune the spectrum of the continuous degrees of freedom to be very soft so as to maximize their interaction with the Ising variables. Specifically we choose the spin-spin interaction

$$
f(\Theta)=z\left\{\cos \Theta+\frac{1}{12} \cos ^{4} \Theta\right\}-(1-z)\left\{\cos ^{2} \Theta-\frac{1}{2} \cos ^{4} \Theta\right\}
$$

which admits collinear ground-state configurations for $z<z_{c}$; the coefficients have been selected so that the first non-vanishing term in a small-angle expansion

$$
f(\Theta) \sim \beta \Theta^{2}+\gamma \Theta^{4}
$$

of (14) is quartic (i.e. $\beta=0$ ) while simultaneously maximizing the value of $z_{c}$. This specific form of the spin-spin interaction (14) leads to very flat free-energy wells with small angular fluctuations described by $<\theta^{2}>\sim T^{\frac{1}{2}}$. We thus expect the presence of these soft continuous degrees of freedom to modify the behavior of the discrete variables of the model. More specifically we hope that they will lead to a significant enhancement of the height stiffness and thus to the possibility of a spin liquid phase (Figure 6). We note that a simpler form of $f(\Theta)$ with $\beta \neq 0$ in (15) results in $<\theta^{2}>\sim T$; this leads to a numerically small

increase 12 of the surface stiffness that does not produce any interesting physical effects. 13 
At $z=0$ the ground-state of the Hamiltonian specified by (12) and (14) is a disordered nematic where the $x y$ variables are ordered but the Ising are not. The finite $z$ term provides an interaction between the discrete variables, and thus their behavior can be described by a fluctuating surface as $T \rightarrow 0$. For $z \neq 0$ there is an additional bond-bond interaction induced by the continuous degrees of freedom; specifically an expansion in the parameter $\frac{z}{T^{\frac{1}{2}}}$ results in the expression

$$
f_{b b}=-\frac{1}{8 T} z^{2} \sigma_{i} \sigma_{j} \sigma_{k} \sigma_{l}\left\langle\left(\theta_{i}-\theta_{j}\right)^{2}\left(\theta_{k}-\theta_{l}\right)^{2}\right\rangle_{I}
$$

where $(i j)$ and $(k l)$ are sites belonging to the same bonds and $I$ refers to the irreducible correlator. Conceptually, this interaction (16) is easier to understand as one between ferromagnetic bonds; if we define

$$
f_{i j}= \begin{cases}1 & \text { if } \sigma_{i} \sigma_{j}=1 \\ 0 & \text { otherwise }\end{cases}
$$

then we can write

$$
\sigma_{i} \sigma_{j} \sigma_{k} \sigma_{l}=4 f_{i j} f_{k l}-f_{i j}-f_{k l}-1
$$

which we insert in (16). Since the total number of FM bonds is fixed in the ground-state manifold by the plaquette constraint, the terms linear in $f_{i j}$ do not depend on the particular bond configuration and thus can be neglected. The bond-bond interaction can then be written as

$$
f_{b b}=-\frac{z^{2}}{32} \sum_{(i j)(k l)} \mathcal{A}_{(i j)(k l)} f_{(i j)} f_{(k l)}
$$

where

$$
\mathcal{A}_{(i j)(k l)}=\frac{1}{T}\left\langle\left(\theta_{i}-\theta_{j}\right)^{2}\left(\theta_{k}-\theta_{l}\right)^{2}\right\rangle_{I}
$$

can be expressed as a bilinear form

$$
\mathcal{A}_{(i j)(k l)}=2 g_{i k}^{2}-4 g_{i k} g_{i l}+\ldots
$$


where

$$
g_{i k} \equiv \frac{2}{T^{\frac{1}{2}}}\left\langle\left(\theta_{i}-\theta_{k}\right)^{2}\right\rangle
$$

is independent of temperature as $T \rightarrow 0$. Numerical evaluation of the constant $\mathcal{A}_{(i j)(k l)}$ indicates that it is maximized by FM bonds with a common vertex that form an angle of $\frac{2 \pi}{3}$, and decays rapidly with increasing bond-bond separation. Therefore this induced bond-bond interaction (19) favors the flat (111) state which has the largest number of such bond configurations. The free energy associated with the entropy of the fluctuating surface $f_{\text {Ising }} \sim T$; since $f_{b b} \sim z^{2}$, at low temperatures it enhances the height stiffness and results in the sequence of transitions (paramagnet, liquid, magnet) discussed above (Figure 6).

\section{THE CANTING TRANSITION}

At low temperatures the spins described by the generalized model of the previous section are energetically unstable in any collinear configuration, and the continuous variables $\theta_{i}$ acquire a small $(\sim z)$ non-zero average value at each site. At $T=0$ a small-angle expansion of the energy about the collinear phase results in the expression

$$
\begin{gathered}
E(\theta)=-\frac{z}{6} \sum_{i j} J_{i j}\left(\theta_{i j}\right)^{2}+h \theta_{i}^{2}+\mathcal{O}\left(\theta_{i j}^{4}\right) \\
J_{i j}=\left(1+3 \sigma_{i} \sigma_{j}\right)
\end{gathered}
$$

where $\theta_{i j} \equiv \theta_{i}-\theta_{j}$ and we have introduced an additional nematic field $h \cos 2 \Theta$ in (12) to "control" the continuous degrees of freedom. For the 100 state each site has exactly two FM bonds, and the lowest eigenvalue of the coupling matrix $J_{i j}$ is zero. By contrast for the 111 phase, where each site has three FM bonds, the matrix $J_{i j}$ has a minimum eigenvalue $\lambda_{\min }=-9$. The lowest eigenvalue of $J_{i j}$ associated with the typical low-temperature state of the height variables is intermediate between these two extreme cases and is always negative.

This canting instability can be illustrated by considering the angular deviation $\left(\theta_{i}\right)$ of a spin at any site $i$ with three AFM bonds, where we maintain spins at all other sites in 
their collinear positions. From (23) we find that the Gaussian energy associated with such an angular deviation is $E=-z\left(\theta_{i}\right)^{2}$; it has a maximum at $\theta_{i}=0$, and therefore the spin at site $i$ is unstable to canting. A higher-order expansion of the energy (23) about the collinear phase results in an effective double-well potential associated with the continuous variables $\theta_{i}$. In the more general case where all the spins are free to cant, the angular deviations on different sites are correlated with a coupling determined by the matrix $J_{i j}$ in (23). At intermediate temperatures where an effective double-well potential develops, the spins are free to fluctuate between its two minima. The "freezing" of each spin in one of these potential wells will occur at a lower temperature, and the entropy associated with this transition is $\ln 2$ per site.

The character of the transition is determined by the coupling matrix $J_{i j}$ which is the sum of two terms, one constant and the other determined by the actual configuration of the surface; the latter can be viewed as the interaction between the canting angles and the height degrees of freedom. The uniform contribution leads to an effective $\phi^{4}$ theory which is in the Ising universality class. This fixed point behavior is stable against perturbative coupling between the continuous and the discrete variables which, by reasons of symmetry, must take the form

$$
F_{\text {int }} \propto(\nabla h)^{2} \theta^{2}
$$

and is irrelevant at long wavelengths. More specifically, an average of (24) over the height fluctuations shifts the transition temperature, and a power-counting argument applied to the renormalization of the $\phi^{4}$-interaction term

$$
\int\left\langle\left(\nabla h_{0}\right)^{2}\left(\nabla h_{r}\right)^{2}\right\rangle d^{2} r \sim \frac{1}{L^{2}}
$$

indicates that it scales to zero at large distances.

In the small-angle expansion of the energy in (23), the interaction between the continuous and the height variables is significant, and thus we cannot exclude the possibility of a firstorder transition or the presence of a new universality class. However numerical studies of this 
model, described below, indicate the stability of the Ising fixed point. As a result, we note that the formation of the canted state has a feedback on the properties of the fluctuating surface. Since the coupling matrix $J_{i j}$ has its lowest minimum eigenvalue for the flat 111 phase, the angular variables have their lowest energy in this configuration and thus modify the $T \rightarrow 0$ ground-state of the discrete variables. In the absence of an intervening first order transition, we thus expect a continuous increase in the stiffness constant $\eta$ which preserves the sequence of three states (paramagnet, liquid, magnet) of the Ising variables discussed above. In Figure 7 we show a schematic of the expected phase diagram; the relative positions of the canting and the liquid phase boundaries must be determined numerically.

\section{NUMERICAL DIAGNOSTICS}

In order to check these ideas we have performed Monte Carlo simulations on the model (14) with $z=0.4$; this is the maximum value for which no traces of $\frac{2 \pi}{3}$ fluctuations were observed in the low-temperature spin configurations $\left(z_{c}=0.56\right.$ for this model). Triangular arrays of $6^{2}, 12^{2}, 24^{2}, 48^{2}$ and $96^{2}$ spins were sequentially cooled from a random configuration at high temperatures with $2.5 \times 10^{5}$ spin flips/site per temperature point, and thermal averages were taken over periods of $2.5 \times 10^{3}$ updates, providing $10^{2}$ approximately independent samples where the distribution of thermodynamic variables was studied. Some measurements (e.g. the surface stiffness coefficient) required longer runs (total number of flips per site $N_{\text {tot }}=10^{7}$ ); in all cases the results were checked for stability with respect to increased number of flips/site. The specific heat results are displayed in Figure 8. At $T_{n e m}=0.25$ there exists a nematic transition into a phase with $<e^{2 i \theta}>\neq 0$, confirmed by the accompanying jump in the spin stiffness shown in of Figure 9 . At $T_{\text {cant }}=0.03$ there is a sharp anomaly in the specific heat that increases with system size. This data excludes the possibility of an incipient first-order transition at low temperatures and thus we expect the sequence of transitions (paramagnet, liquid, magnet) described schematically in Figures 6 and 7 . 
We associate the sharp structure in the specific heat data at low temperatures (Figure 8) with the canting instability. A standard finite-size analysis (see Figure 10) confirms that this transition is in the Ising universality class. Briefly for a finite system of size $L$ that is smaller than the relevant correlation length $\xi$ the specific heat scales as

$$
c_{v} \sim L^{\frac{\alpha}{\nu}} f\left(\epsilon L^{\frac{1}{\nu}}\right)
$$

where $\alpha$ and $\nu$ are the exponents associated with $c_{v}$ and $\xi$ in the limit $L \rightarrow \infty$, and $\epsilon \equiv\left(\frac{T-T_{c}}{T_{c}}\right)$ is the reduced temperature; here $f(x)$ is a universal function. For the case of the $2 D$ Ising ferromagnet $\alpha=0$ and $\nu=1$ so that (26) becomes

$$
c_{v}=A \log L+f(\epsilon L)
$$

where

$$
\lim _{\epsilon L \rightarrow \infty} f(\epsilon L)=-A \log (\epsilon L)
$$

ensuring that the $2 D$ Ising results are recovered in the limit of infinite system-size. In Figure 10 we have displayed the universal function $f(x)$ in (27) as a function of $\log (\epsilon L)$ for five system sizes; the resulting linear plot confirms the Ising values of $\alpha$ and $\nu$. Furthermore we vary the coefficient $A$ in (27) to get the best fit to the numerical data; its optimal value $(A=0.50)$ is equal to the slope of the linear plot in Figure 9, consistent with (28). This coefficient $A$ is the amplitude associated with the specific heat at the transition, and is consistent with the known value $\left(A_{\text {Ising }}=0.53\right)$ for the two-dimensional Ising ferromagnetic model.14 A crude integration of the specific heat peak at $T_{\text {cant }}$ in Figure 8 leads to an estimated entropy $S \sim 0.85 \ln 2$; both these amplitude and the entropy results are consistent with approximately one Ising degree of freedom/spin at the transition.

In order to confirm that the $T_{\text {cant }}=0.03$ transition is due to the presence of the continuous degrees of freedom, we apply a nematic field $h_{n e m}=h \cos 2 \theta_{i}$ to suppress their thermal fluctuations. The specific heat anomaly remains sharp (Figure 11a) indicating that this field $h_{n e m}$ is not conjugate to the order parameter; however it does affect the transition 
temperature as shown in the resulting phase diagram in Figure 11b. We can estimate the critical nematic field strength $\left(h_{\text {crit }}\right)$ from the quadratic form of the energy; it is the minimum value of $h$ which maintains the expression (23) positive definite. For a typical state of Ising variables at $T=0.03$, we have diagonalized the coupling matrix $J_{i j}$ and found that $\lambda_{\text {min }}=-7.5$. Thus the transition is completely suppressed if $h_{n e m} \geq 0.5$ in rough agreement with the numerical results (Figure 11), where we were not able to follow the phase line down to temperatures $T<0.015$ due to problems of equilibration.

We can also estimate the canting transition temperatures using the quartic terms in the small-angle expansion of the energy (23). For this purpose we consider again the canting instability of a spin at site $i$ with three AFM bonds, where again we maintain all its neighbors in their collinear positions. The renormalized energies associated with AFM and FM bonds are

$$
\begin{aligned}
& E_{A F M}^{R} \approx \theta_{i j}^{2}\left(\frac{1}{3} z+\left\langle\theta_{i j}^{2}\right\rangle\right) \\
& E_{F M}^{R} \approx \theta_{i j}^{2}\left(-\frac{2}{3} z+\left\langle\theta_{i j}^{2}\right\rangle\right)
\end{aligned}
$$

so that the spin at site $i$ becomes unstable at

$$
\frac{1}{6} z \approx\left\langle\theta_{i j}^{2}\right\rangle=\frac{T}{\rho_{s}(T)}
$$

where $\rho_{s}(T)$ is the spin stiffness; (30) is the defining equation for the canting transition temperature. Our measured quantities

$$
\frac{T_{c}}{\rho_{s}\left(T_{c}\right)} \approx 0.06 \approx \frac{z}{6}
$$

are consistent with the expression (30).

In order to characterize the fluctuations of the Ising degrees of freedom, we determine the height surface by measuring the relative height fluctuations $\left\langle h_{r}^{2}\right\rangle \equiv\left\langle\left(h^{2}(r)\right\rangle-\langle h(r)\rangle^{2}\right.$ between the middle and the edge of each array. We observe that for $T<T_{n e m}$ the height fluctuations are described by the $T=0$ fixed point of the pure Ising antiferromagnet; the logarithmic behavior of the fluctuations, shown in Figure 12, is only robust up to the average distance between height defects $\left(\xi \sim 3 \times 10^{3}\right)$ which exceeds all system sizes studied. As expected, 
the height stiffness $\left(\left\langle h_{r}^{2}\right\rangle=\frac{\ln L}{\eta \pi}\right)$ increases in the approach to the canting transition; this behavior is also displayed in the profile of the height fluctuations as a function of decreasing temperature (Figure 13). Unfortunately we cannot maintain the discrete degrees of freedom in equilibrium to low enough temperatures to directly observe the presence of the height liquid. However, since the specific heat data indicates no first-order transition, the surface stiffness must increase continuously; furthermore we know, from our arguments about the canting instability, that the surface will become spontaneously oriented with $\eta>\eta_{c 2}$ in the limit $T \rightarrow 0$. Our numerical results confirm the expected enhancement of the surface stiffness coefficient with decreasing temperature. We therefore infer the presence of a finitetemperature fluctuating surface with no defects; its magnetic analogue is a spin liquid with power-law Ising correlations.

Thus we observe that in the vicinity of the canting transition, the surface stiffness is enhanced. We expect that for $T<T_{\text {cant }} \eta$ will increase above its critical value $\left(\eta_{c 1}\right)$, resulting in a finite-temperature height liquid on all length scales. However on our length-scales of measurement even the Ising system is a liquid, since at $T=0.03$ its correlation length is several thousand lattice spacing, well beyond the largest system sizes studied. We have used the surface stiffness to characterize the low-temperature behavior of the generalized model and to distinguish it from its discrete counterpart.

The dynamics of the resulting liquid, even at short length-scales, is also very different from that of the pure Ising case due to the interaction between its discrete and continuous degrees of freedom. In order to illustrate this feature, we consider small changes to our fluctuating surfaces associated with the addition/subtraction of an elementary block. For the pure Ising case there is no energy cost associated with this process; however it changes the structure of the coupling matrix $J_{i j}$ in (23), and results in a dramatic reconstruction of the continuous degrees of freedom. As $T \rightarrow T_{\text {cant }}$ we expect that the canting instability will appear first in the lowest eigenmodes $J_{i j}$, so that we can neglect all the others. In Figure 14 we display a typical surface and its lowest eigenmode is shown in Figure 15. The subtraction 
of a cube not only rearranges the energy level ordering but also modifies each state. In order to illustrate this property, we subtract a single block from the surface displayed in Figure 14 at the location denoted by the arrow; we then rediagonalize the coupling matrix and display the eigenmode that has the maximum overlap with that shown in Figure 15 . The dramatic reconstruction of the continuous degrees of freedom displayed by comparison of Figures 15 and 16 requires some time; in the transient regime the energy of the angular degrees of freedom is high implying the presence of macroscopic barriers associated with this local process.

The preceeding discussion suggests a distribution of surface relaxation times. 16,17 We thus measure the time-dependent overlap

$$
Q(t)=\frac{1}{N} \sum_{i=1}^{N}(-1)^{h_{i}(t)-h_{i}(0)}
$$

between initial and final Ising states at a given temperature, probing the system's exploration of phase space ; any feature in $Q(t)$ indicates memory on the time-scale of those structure. 18 Before presenting our results, let us pause briefly to discuss the possible scenarios. In a hightemperature paramagnet ("gas") the exponential spatial and temporal spin correlations lead to an exponential decay of the overlap. By contrast, in a spin liquid the decay of $Q(t)$ is power-law; this is the situation for the $T \rightarrow 0$ Ising triangular antiferromagnet, and is maintained for this discrete case at any finite temperature up to the length-scale defined by the defect-defect separation. In a glass the functional form of the overlap decay is yet slower than power-law due to the broad distribution of relaxation times. A slow decay of the overlap on a time-scale $\tau$ indicates the presence of barriers of height $\sim T \ln \tau$, and the magnitude of $Q(\tau)$ measures the number of metastable states separated by these barriers. For example, the Sherrington-Kirkpatrick (SK) spin glass, where the interaction is infinite-range, has a minimum correlation volume that scales with the system-size $(L)$; the associated overlap decay indicates that both the barrier number and their maximum height scale as powers of L. By contrast, the Edwards-Anderson spin glass with its short-ranged interaction, displays a broad distribution of length-scales; the associated barrier distribution is continuous and 
only limited by $L$, where the number of barriers with maximum height scales as $L^{\alpha}$ where $\alpha>0$.

In Figures 17 and 18 we show our results for the time-dependent overlap of the generalized model above and below the canting transition; the measurements are averaged over different time-scales. In Figure 17 we observe that the short-time behavior of $[Q(t)]^{2}$ is qualitatively similar for $T>T_{\text {cant }}$ and $T<T_{\text {cant }}$; both display similar features at these time-scales but are different from the pure Ising case where this $[Q(t)]^{2}$ is completely flat (dotted line in Figure 17). At longer times (Figure 18) only the overlap measured at $T<T_{\text {cant }}$ has discernible structure; $[Q(t)]^{2}$ for $T>T_{\text {cant }}$ on these time-scales is featureless. Figure 18a indicates that for $T<T_{\text {cant }}$ the system is making transitions between a few metastable states separated by large barriers of similar height; their size is proportional to the logarithm of the pseudo-period of the observed undulations. This data also suggests that there is a minimum correlation volume associated with this generalized model; presumbably it is related to the number of sites involved in the reconstruction of the $\theta_{i}$ variables following an elementary process. A study of the identical overlap for a larger system-size is necessary to determine the finite-size scaling of the maximum barrier height and number; in particular we would like to investigate whether the system becomes "localized" in phase space thereby breaking ergodicity. The results displayed in Figure 18b indicate that the barrier heights do increase for larger $L$; however

$$
\lim _{t \rightarrow \infty}[Q(t)]^{2}=\lim _{t \rightarrow \infty}\left[Q_{\text {Ising }}(t)\right]^{2}
$$

so that the number of metastable states does not increase with system-size and the minimum correlation volume remains finite. Thus, though the system retains some long-time memory of its initial Ising state, it is a "slow" viscous liquid and not a glass; this is because in the thermodynamic limit $(L \rightarrow \infty)$ it can explore almost all of phase space and thus is effectively ergodic. 


\section{DISCUSSION}

In summary we have constructed a frustrated spin model whose discrete degrees of freedom acquire power-law correlations at finite temperatures. There is a true thermodynamic transition into this "spin liquid", which exists as an intermediate phase separating the paramagnetic ("gas") and the magnetic ("solid") states. This spin liquid has an extensive zero-point entropy and is the finite-temperature analogue of the $T \rightarrow 0$ phase of the Ising antiferromagnet on the triangular lattice. This highly degenerate ground-state manifold is conveniently parametrized by an undulating surface, whose fluctuations on large lengthscales are characterized by a surface stiffness constant. For the purely discrete model, the mapping between the spin and the membrane problem is no longer uniquely defined at any finite temperature due to the presence of singularities in the surface.

In this paper we have constructed an $x y$ spin model on the triangular lattice that has both continuous and Ising low-energy excitations; the ground-state configurations of these discrete variables are isomorphic to those of the pure Ising antiferromagnet above. In the generalized model, the interaction between the $x y$ and the Ising degrees of freedom results

in the continuous enhancement of the surface stiffness with decreasing temperature. As a result, the defects are bound at finite temperatures and the surface mapping becomes well-defined. For very low temperatures the stiffness coefficient becomes sufficiently large to spontaneously orient the surface, and the ground-state is unique.

We thus expect a sequence of three phases (paramagnet, liquid, magnet) associated with the discrete variables as a function of decreasing temperature. There is also a canting instability where the angular deviations $\theta_{i}$ acquire a non-zero average at each site; the locations of these canting and liquid phase boundaries must be determined numerically. Similarly the possibility of an intervening first-order transition cannot be excluded analytically. Therefore we have performed Monte Carlo simulations on the generalized $x y$ model described here. At low temperatures $\left(T_{\text {cant }}=0.03\right.$ for $\left.z=0.4\right)$ we observe a sharp anomaly in the specific heat which we attribute to the canting transition; a finite-size scaling analysis of these results 
indicates that it is in the Ising universality class. Application of a nematic field suppresses $T_{\text {cant }}$, confirming that it is due to the continuous degrees of freedom. The resulting phase line and the measured values of $T_{\text {cant }}$ and $\rho_{s}\left(T=T_{\text {cant }}\right)$ are all consistent with that expected from a small-angle expansion of the energy. Measurement of the surface stiffness coefficient confirms its enhancement with decreasing temperature, though unfortunately equilibration problems prevent the direct observation of the height liquid when $\eta>\eta_{c 1}$. The interaction between the discrete and the continuous variables also leads to the presence of macroscopic barriers associated with local processes. In order to probe the systems exploration of phase space, we measure the overlap between the final and initial Ising states averaged over different time-scales; we find that though the system has some long-term memory it remains effectively ergodic in the thermodynamic limit.

More generally, we would like to emphasize that the classical liquid discussed here is not an anomalous result restricted to the specific Hamiltonian constructed above. In the broadest terms, the stability of such a finite-temperature phase with power-law discrete spin correlations could emerge from any model with the following crucial features:

(i) At low temperatures it should have both discrete and continuous spin excitations

(ii) Its discrete degrees of freedom retain an extensive entropy 15 at $T \rightarrow 0$ and, in all known examples in finite-dimensions, the associated ground-state manifold is conveniently described by a freely fluctuating surface

(iii) The interaction between the discrete and the continuous degrees of freedom should enhance the stiffness constant of the associated surface

We note, for example, that an $x y$ model on the kagomé lattice satisfies all three of these conditions; furthermore since $\eta_{P o t t s}=\eta_{c 1}$ we expect that a weak interaction between the continuous and discrete degrees of freedom should be sufficient to drive the system into a liquid phase.

The enhanced stiffness constant associated with the model specified by criteria $(i),(i i)$ 
and (iii) not only affects the equilibrium properties of the underlying discrete degrees of freedom, but modifies their dynamics as well. In particular a local process, which in the purely discrete model has a negligible energy cost, can now involve significant rearrangement of the continuous degrees of freedom; this surface reconstruction is not instantaneous and thus there will be a transient regime where the surface energy is large. The distribution of the associated barriers can be probed by a study of the overlap decay between final and initial discrete states. For the specific model studied in this paper, we find that there is a large minimum correlation volume that remains finite in the thermodynamic limit resulting in a viscous spin liquid that effectively retains its ergodicity. Though the initial Hamiltonian has purely local couplings, the interplay between the Ising and the $x y$ degrees of freedom generates long-range interactions between discrete spins on a length-scale that is large but finite in the limit $L \rightarrow \infty$. The development of a glass requires the generation of an infiniterange correlation; this may be difficult to achieve in two dimensions due to the large phase space available to long-wavelengh spin fluctuations. It is difficult to construct an analogous model in higher dimensions since the tendency towards magnetic ordering is larger than in $d=2$, and thus it will be difficult to find a discrete system that retains its extensive entropy in the limit $T \rightarrow 0$ and thus to satisfy $(i i)$. However, if found, we expect it to have still slower dynamics and even possible glassiness in the absence of disorder.

We are very grateful to B. Doucot and L. Levitov for sharing their ideas and insights with us as this work progressed, and to M.V. Feigelman for a critical reading of the manuscript. The numerical work was performed on an NEC SX-3 at the HNSX Supercomputer Center (Woodlands, Texas), and we thank N. Trouillier for assistance with the code. P. Coleman acknowledges financial support from NSF Grant No. DMR-89-13692. 


\section{REFERENCES}

${ }^{1}$ G.H. Wannier, Phys. Rev. B 79, 357 (1950); R.M.F. Houtappel, Physica 16, 425 (1950).

${ }^{2}$ J. Stephenson, J. Math. Phys., 5, 1009 (1967).

${ }^{3}$ H.W.J. Blote and H.J. Hilhorst, J. Phys. A.: Math. Gen., 15, L631 (1982); B. Nienhuis, H.J. Hilhorst and H.W.J. Blote, J. Phys. A.: Math. Gen.17, 3559 (1984).

${ }^{4}$ P.W. Anderson, Phys. Rev. 102, 1008 (1956).

${ }^{5}$ X. Obradors, A. Labarta, A. Isalgue, J. Tejada, J. Rodridgues and M. Pernet, Sol. Stat. Comm., 65189 (1988).

${ }^{6}$ C. Broholm, G. Aeppli, G.P. Espinosa and A.S. Cooper, Phys. Rev. Lett. 65, 3173 (1990); G. Aeppli, C. Brohom, A.P. Ramirez, G.P. Espinosa and A.S. Cooper, J. of Mag. and Mag. Mat.,90-91 255 (1990).

${ }^{7}$ A.P. Ramirez, G.P. Espinosa and A.S. Cooper, Phys. Rev. Lett. 64, 2070 (1990).

${ }^{8}$ P. Chandra and P. Coleman in Les Houches Lecture Notes 1991, ed. B. Doucot and J. Zinn-Justin, to appear.

${ }^{9}$ A.P. Ramirez in Annual Review of Mat. Science, ed. E.N. Kaufmann, to appear.

${ }^{10}$ For a review of this phenomenon see J.M. Kosterlitz and D.J. Thouless in Progress in Low-Temperature Physics,ed. D.F. Brewer (North-Holland, Amsterdam, 1978).

${ }^{11}$ P. Nozieres and F. Gallet, J. Physique, 48, 353 (1987).

${ }^{12}$ We determined the magnitude of this prefactor using a Monte Carlo simulation.

13 This is the spirit of the approach taken in D.H. Lee, G. Grinstein and J. Toner, Phys. Rev. Lett., 56, 2318, (1986); these authors have neglected the linear temperature dependence of the surface stiffness and thus it is improbable that there will be a liquid phase in their model. 
14 e.g. see K. Huang, Statistical Mechanics (J. Wiley, New York) 1987.

${ }^{15}$ In the absence of an extensive discrete entropy, thermal fluctuations will always select the flat state. More specifically the failure of "order from disorder" will only occur if $S_{D}>S_{\theta}$ where $S_{D}$ and $S_{\theta}$ are the discrete and continuous entropic contributions respectively, as discussed in P. Chandra and B. Doucot, J. Phys. A: Math. Gen. (in press) .

${ }^{16}$ J.A. Hertz, L. Fleishmann and P.W. Anderson, Phys. Rev. Lett. 43, 942, 1982.

${ }^{17}$ L.B. Ioffe and M.V. Feigelman, Sov. Phys. JETP, 62, 376 (1985).

18 e.g. Ph. Refregier, E. Vincent, J. Hammann and M. Ocio, J. Physique, 48, 1533, 1987; E. Vincent, J. Hammann and M. Ocio in Recent Progress in Random Magnets, ed. D.H. Ryan (McGill University, Montreal, 1991). 


\section{FIGURES}

FIG. 1. A section of the (111) ground-state configuration of the Ising triangular model a) before and b) after the $\pi$-flip of a spin (bold) with exactly three AFM bonds (solid lines); all other circled spins can be similarly reversed at zero energy cost resulting in a lower bound for the $T \rightarrow 0$ entropy of $S \geq \frac{N}{3} \ln 2$.

FIG. 2. A section of the (100) ground-state configuration of the Ising triangular model a) before and b) after the $\pi$-flip of a spin (bold) with four AFM bonds (solid lines); this process creates two "bad" triangles (shaded) whose subsequent separation by an additional spin-flip, illustrated in c), does not further violate the plaquette constraint.

FIG. 3. A schematic illustrating that the energy cost associated with two defects in the Ising triangular model is independent of their relative position; here only the AFM bonds are displayed, and the dotted lines refer to the bonds "flipped" in moving a defect from its location in a) to that in b).

FIG. 4. A section of a typical ground-state Ising configuration (a) with its corresponding rhombi tiling (b) and its associated surface representation (c) where the heights $\left(h_{i}\right)$ are indicated.

FIG. 5. The height differences around a closed loop (bold line with arrows) in the a) the absence and b) the presence of a height defect (dotted lines indicate the bonds "flipped" to create the defect pair); note that this loop sum is zero if the number of defects enclosed is even but is a multiple of six if it is odd.

FIG. 6. Schematic of the sequence of paramagnet-liquid-magnet transitions expected as a function of increasing height stiffness constant.

FIG. 7. Proposed phase diagram for the generalized spin model described in the text where $\mathrm{C}, \mathrm{L}$ and $\mathrm{S}$ refer to the canted, liquid and solid phases repectively and the dotted line indicates a first-order transition . 
FIG. 8. Specific heat results for the $z=0.4$ model described in the text for arrays of $6^{2}, 12^{2}$, $24^{2}, 48^{2}$ and $96^{2}$ spins.

FIG. 9. Spin Stiffness Results for the $z=0.4$ model described in the text for arrays of $6^{2}, 12^{2}$, $24^{2}, 48^{2}$ and $96^{2}$ spins.

FIG. 10. Finite-size Scaling Analysis for the Specific Heat Data $T<T_{c}$ where $T_{c}=0.03$; the linear behavior of this plot confirms that the associated canting transition is in the Ising universality class (similar results are obtained for the $T>T_{c}$ case).

FIG. 11. a) Specific heat data for $h_{n e m}=0.0,0.3$ indicating that the anomaly remains sharp despite the suppression of $T_{c}$ and b) Phase diagram of $h_{n e m}$ vs $T_{I s i n g}$ where $h_{n e m}$ is an applied nematic field (see text).

FIG. 12. $\left\langle h_{r}^{2}>\right.$ vs. $\ln L$ where the height fluctuations $\left\langle h_{r}^{2}\right\rangle \equiv\left\langle h^{2}(r)\right\rangle-\langle h(r)\rangle^{2}$ are related to the height stiffness $(\eta)$ and the array length $(L)$ by the expression $<h_{r}^{2}>=\frac{\ln L}{\eta \pi}$.

FIG. 13. Profile of height fluctuations across the diagonal of a $96 \times 96$ array as a function of decreasing temperature.

FIG. 14. A snapshot of a "typical" surface in the vicinity of the canting transition; the arrow points to one location where the addition/subtraction of an elementary cube can occur.

FIG. 15. The lowest eigenmode $(|\Psi|)$ of the "typical" state in Fig. 14 in the vicinity of the canting transition

FIG. 16. An eigenmode corresponding to a state after the subtraction of an elementary building block in Fig. 14 at the location denoted by the arrow; the eigenmode displayed here has a maximal overlap with the original one shown in Fig. 15. 
FIG. 17. The time-dependent height overlap $[Q(t)]^{2}=\left(\frac{1}{N_{i}} \sum_{i}(-1)^{\left\{h_{i}(t)-h_{i}(0)\right\}}\right)^{2}$ averaged over a) 10 and b) 100 Monte Carlo steps for a $24^{2}$ array; its behavior for $T>T_{c}$ and $T<T_{c}$ is qualitatively similar.

FIG. 18. The time-dependent height overlap $[Q(t)]^{2}=\left(\frac{1}{N_{i}} \sum_{i}(-1)^{\left\{h_{i}(t)-h_{i}(0)\right\}}\right)^{2}$ averaged over 1000 Monte Carlo steps for a a) $24^{2}$ and a b) $48^{2}$ array; the pseudo-periodic structure implies the presence of large barriers whose height but not number increase with system size and thus the system is effectively ergodic in the thermodynamic limit. 